\title{
ALGEBRAIC ORIENTED COHOMOLOGY THEORIES
}

\author{
ALEXANDER MERKURJEV
}

\begin{abstract}
For every smooth projective variety over an infinite field $F$ we define its fundamental polynomial in $\mathbb{Z}[\mathbf{b}]=\mathbb{Z}\left[b_{1}, b_{2}, \ldots\right]$ and prove that the fundamental polynomials generate the Lazard ring Laz $\subset \mathbb{Z}[\mathbf{b}]$. Using description of invariant prime ideals in Laz, due to Landweber, we assign to every smooth projective variety $X$ the numbers $n_{p}(X)$ for every prime integer $p$. Inequality $n_{p}(Y)>n_{p}(X)$ for some prime $p$ is an obstruction for existence of a morphism $Y \rightarrow X$ over $F$.
\end{abstract}

\section{INTRODUCTION}

Let $\operatorname{Sm}(F)$ be the category of smooth quasi-projective varieties over a field $F$. M. Levine and F. Morel have defined in [7] an oriented cohomology theory over $F$ as a contravariant functor $A^{*}$ from the category $\operatorname{Sm}(F)$ to the category of graded commutative rings satisfying certain properties (see section 2). Examples of the oriented cohomology theories are $K^{*}$ given by the Grothendieck rings of varieties in $\operatorname{Sm}(F)$ (Example 2.2) and $H^{*}$ given by the Chow rings (Example 2.1).

It is proved in [7] that if char $F=0$ (resolution of singularities is used) then there exists a universal oriented algebraic cobordism cohomology theory $\Omega^{*}$. For every oriented cohomology theory $A^{*}$ there is unique morphism of cohomology theories $\Omega^{*} \rightarrow A^{*}$ commuting with the push-forward homomorphisms. For a variety $X \in \operatorname{Sm}(F)$ the group $\Omega^{*}(X)$ is generated by the classes $[f]$ corresponding to projective morphisms $f: Y \rightarrow X$ in $\operatorname{Sm}(F)$. The homomorphism $\Omega^{*}(X) \rightarrow A^{*}(X)$ takes the class $[f]$ to $f_{A}\left(1_{Y}\right)$, where $f_{A}$ is the push-forward homomorphism in $A^{*}$. Thus, the image of the morphism $\Omega^{*}(X) \rightarrow A^{*}(X)$, which we denote by $A_{c}^{*}(X)$, can be defined just in terms of the theory $A$ : the group $A_{c}^{*}(X)$ is generated by the elements $f_{A}\left(1_{Y}\right)$ for all projective morphisms $f: Y \rightarrow X$.

To every oriented cohomology theory $A$ one has associated a commutative formal group law $\Phi^{A}$ over the coefficient ring $A^{*}(\mathrm{pt})$. The formal group law $\Phi^{\Omega}$ is the universal one and the coefficient ring $\Omega^{*}(\mathrm{pt})$ is the Lazard ring.

In the present paper we consider oriented cohomology theories on $\operatorname{Sm}(F)$ for infinite fields $F$ of arbitrary characteristic and don't refer to the problem of resolution of singularities and existence of the cobordism theory. The idea is to consider "large" oriented cohomology theories $A^{*}$ such that the natural

Date: December, 2001.

Partially supported by the N.S.F. 
homomorphism $\Omega^{*}(X) \rightarrow A^{*}(X)$ is injective at least for $X=$ pt and work inside $A^{*}$ instead of $\Omega^{*}$.

How to construct "large" cohomology theories? In section 4 we consider an operation (we call it tilde operation), which assigns to every oriented cohomology theory $A^{*}$ another theory $\widetilde{A}^{*}$ defined by

$$
\widetilde{A}^{*}(X)=A^{*}(X) \otimes \mathbb{Z}[\mathbf{b}]=A^{*}(X)[\mathbf{b}]
$$

where $\mathbb{Z}[\mathbf{b}]=\mathbb{Z}\left[b_{1}, b_{2}, \ldots\right]$ is the polynomial ring in infinitely many variables. We define the push-forward homomorphisms in $\widetilde{A}^{*}$ in such a way that the inverse Todd genus of the natural embedding $A^{*} \hookrightarrow \widetilde{A}^{*}$ is the universal one (with the coefficient being the indeterminates $b_{i}$ ). We prove that the two theories $\widetilde{H}^{*}$ and $\widetilde{K}^{*}$ are large enough so that the coefficient rings $\widetilde{H}_{c}^{*}(\mathrm{pt})$ and $\widetilde{K}_{c}^{*}(\mathrm{pt})$ are both isomorphic to the Lazard ring. In sections 6 and 7 we follow closely the method of [10].

For every projective variety $X \in \mathbf{S m}(F)$ we define the fundamental polynomial $\mathbf{F}_{X}^{H} \in \mathbb{Z}[\mathbf{b}]$ and prove that for any infinite field $F$ the fundamental polynomials of all projective $X \in \operatorname{Sm}(F)$ generate the same ring - the Lazard ring Laz considered as a subring of $\mathbb{Z}[\mathbf{b}]$. The fundamental polynomials $\mathbf{F}_{X}^{H}$ do not change under field extensions (and therefore can computed over an algebraic closure of $F$ ); nevertheless, they keep track of an arithmetic information on $X$. Namely, all the coefficients of $\mathbf{F}_{X}^{H}$ are divisible by the greatest common divisor of the degrees $[F(x): F]$ of all closed points of $X$. For example, existence of division algebras of a given dimension over an extension of $F$ explains the fact that the fundamental polynomial of the projective space $\mathbb{P}_{F}^{n}$ is divisible by $n+1$ in $\mathbb{Z}[\mathbf{b}]$ (Example 3.7 ), the well known fact in topology (see $[9, \mathrm{Ch}$. $\mathrm{VII}])$.

In section 9 we prove that characteristic classes of vector bundles over $X \in$ $\operatorname{Sm}(F)$ take values in the subgroup $A_{c}^{*}(X) \subset A^{*}(X)$. We use this result in section 10 where we study the Landweber-Novikov operations on Laz. In section 11 we introduce ideals $I(X) \subset$ Laz for every projective variety $X \in$ $\operatorname{Sm}(F)$, consisting of the fundamental polynomials of all projective varieties $Y \in \mathbf{S m}(F)$ such that there is a morphism $Y \rightarrow X$ (defined over $F$ ). We prove that the ideal $I(X)$ is invariant under the Landweber-Novikov operations and so are all the associated prime ideals. Invariant prime ideas were described by Landweber in [5]. Based on this description one can associate to every projective variety $X \in \mathbf{S m}(F)$ and every prime integer $p$ an integer $n_{p}(X) \in$ $\{0,1, \ldots, \infty\}$. Inequality $n_{p}(Y)>n_{p}(X)$ for some prime $p$ is an obstruction for existence of a morphism $Y \rightarrow X$ defined over $F$.

Although the paper is purely algebraic, the most of the constructions are borrowed from topology. The class $\left[-T_{X}\right] \in K_{0}(X)$ of the tangent bundle $T_{X}$ over $X$ is a replacement for the stable normal bundle of $X$. The tilde operation is analogous to the wedge product with the Thom spectrum $M U$. The embedding of the Lazard ring into $\mathbb{Z}[\mathbf{b}]$ is the Hurewicz homomorphism 
$\pi_{*}(M U) \rightarrow H_{*}(M U)$. The Landweber-Novikov operations are induced by those on the spectrum $M U$.

\section{Definition of AN ORIEnted COHOMOlogy THEORY}

Let $F$ be a field, $\operatorname{Sm}(F)$ the category of smooth quasi-projective varieties over $F$. Let $A^{*}$ be a functor from $\operatorname{Sm}(F)^{o p}$ to the category GrRings of $\mathbb{Z}$-graded commutative rings. For a morphism $f: Y \rightarrow X$ in $\operatorname{Sm}(F)$ the (pull-back) ring homomorphism $A^{*}(f)$ is denoted by $f^{A}$.

An oriented cohomology theory over $F$ (see [7]) is a functor

$$
A^{*}: \operatorname{Sm}(F)^{o p} \rightarrow \text { GrRings }
$$

together with a graded (push-forward) group homomorphism

$$
f_{A}: A^{*}(Y) \longrightarrow A^{*+d}(X)
$$

for every projective morphism $f: Y \rightarrow X$ in $\operatorname{Sm}(F)$ of pure codimension $d$, satisfying the following:

(i) For a pair of projective morphisms $f: Y \rightarrow X$ and $g: Z \rightarrow Y$, one has $(f \circ g)_{A}=f_{A} \circ g_{A}$.

(ii) Let $E \rightarrow X$ be a vector bundle over $X \in \operatorname{Sm}(F)$ of rank $r, \mathbb{P}(E) \rightarrow X$ associated projective bundle. Then $A^{*}(\mathbb{P}(E))$ is a free $A^{*}(X)$-module with basis $1, \xi, \xi^{2}, \ldots, \xi^{r-1}$, where $\xi=s^{A} s_{A}\left(1_{\mathbb{P}(E)}\right)$, and $s$ is the zero section of the tautological line bundle over $\mathbb{P}(E)$.

(iii) Let

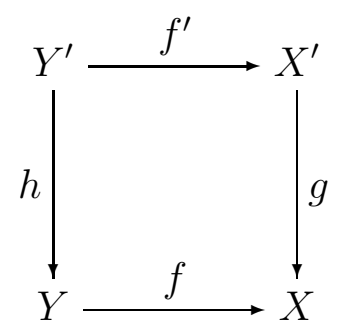

be a transverse Cartesian square in $\operatorname{Sm}(F)$ with $f$ a projective morphism, i.e. the sequence of tangent bundles over $Y^{\prime}$

$$
0 \longrightarrow T_{Y^{\prime}} \stackrel{d f^{\prime}+d h}{\longrightarrow} f^{*} T_{X^{\prime}} \oplus h^{*} T_{Y} \stackrel{d g-d f}{\longrightarrow}(f h)^{*} T_{X} \longrightarrow 0
$$

is exact. Then $f_{A}^{\prime} \circ h^{A}=g^{A} \circ f_{A}$.

(iv) Let $p: V \rightarrow X$ be an affine bundle (a torsor for a vector bundle over $X$ ). Then $p^{A}: A^{*}(X) \rightarrow A^{*}(V)$ is an isomorphism.

(v) (Projection formula) Let $f: Y \rightarrow X$ be a projective morphism in $\operatorname{Sm}(F)$. Then for every $a \in A^{*}(X)$ and $b \in A^{*}(Y), f_{A}\left(b \cdot f^{A}(a)\right)=f_{A}(b) \cdot a$.

The ring $A^{*}(\mathrm{pt})$, where pt $=\operatorname{Spec}(F)$, is called the coefficient ring of $A^{*}$. For every $X \in \mathbf{S m}(F), A^{*}(X)$ is an algebra over $A^{*}(\mathrm{pt})$. 
Example 2.1. ([7, Ex. 1.2]) The Chow cohomology theory $H^{*}$ assigns to every variety $X \in \operatorname{Sm}(F)$ the Chow ring $H^{*}(X)=\mathrm{CH}^{*}(X)$. The push-forward and pull-back homomorphisms are defined in [2]. The coefficient ring $\mathrm{CH}^{*}(\mathrm{pt})$ is equal to $\mathbb{Z}$.

Example 2.2. ([7, Ex. 1.3]) The $K$-theory assigns to every variety $X \in \mathbf{S m}(F)$ the Laurent polynomial ring $K^{*}(X)=K_{0}(X)\left[t, t^{-1}\right]$ graded by $\operatorname{deg}(t)=-1$, i.e. $K^{i}(X)=K_{0}(X) t^{-i}$. If $f: Y \rightarrow X$ is a projective morphism of pure codimension $d$, then for every $a \in K^{*}(Y), f_{K}\left(a t^{-i}\right)=f_{*}(a) t^{-i-d}$, where $f_{*}$ is the push-forward homomorphism in algebraic $K$-theory. We have also $K^{*}(\mathrm{pt})=\mathbb{Z}\left[t, t^{-1}\right]$.

Let $p: X \rightarrow \mathrm{pt}$ be the structure morphism. If $X$ is projective of dimension $d$, we define the fundamental class $[X]^{A}$ of $X$ in the theory $A$ as the element

$$
[X]^{A}=p_{A}\left(1_{X}\right) \in A^{-d}(\mathrm{pt}) .
$$

For example, $[\mathrm{pt}]^{A}=1,[X]^{H}=0$ if $d>0$ and $[X]^{K}=t d(X) t^{d}$, where $t d(X)=p_{*}\left(\left[\mathcal{O}_{X}\right]\right) \in \mathbb{Z}$ is the Todd number of $X[2$, Example 15.2.13].

Proposition 2.3. Let $X$ and $Y$ be projective varieties in $\operatorname{Sm}(F)$. Then $[X \times Y]^{A}=[X]^{A} \cdot[Y]^{A}$.

Proof. Consider Cartesian transverse square

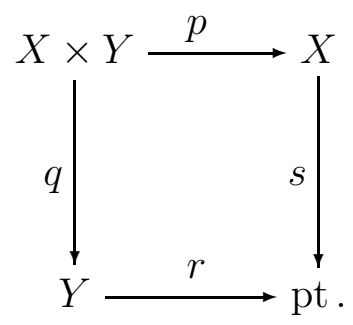

We have

$$
\begin{aligned}
{[X \times Y]^{A} } & =(s p)_{A}\left(1_{X \times Y}\right) \\
& =\left(s_{A} p_{A} q^{A}\right)\left(1_{Y}\right) \quad(\text { property }(\mathrm{iii})) \\
& =\left(s_{A} s^{A} r_{A}\right)\left(1_{Y}\right) \quad(\text { projection formula) } \\
& =s_{A}\left(1_{X}\right) \cdot r_{A}\left(1_{Y}\right) \\
& =[X]^{A} \cdot[Y]^{A}
\end{aligned}
$$

For every smooth variety $X$ consider the graded subgroup $A_{c}^{*}(X)$ in $A^{*}(X)$ generated by the elements $f_{A}\left(1_{Y}\right)$ for all projective morphisms $f: Y \rightarrow X$ in $\operatorname{Sm}(F)$. Clearly, $A_{c}^{i}(X)=0$ if $i>\operatorname{dim}(X)$. For a projective morphism $g: X \rightarrow X^{\prime}$ the push-forward map $g_{A}$ takes $A_{c}^{*}(X)$ to $A_{c}^{*}\left(X^{\prime}\right)$.

The subgroup $A_{c}^{*}(\mathrm{pt}) \subset A^{*}(\mathrm{pt})$ is generated by the fundamental classes $[X]^{A}$ for all smooth projective varieties $X$. Proposition 2.3 shows that $A_{c}^{*}(\mathrm{pt})$ is a subring in $A^{*}(\mathrm{pt})$. 
Example 2.4. $H_{c}^{*}(\mathrm{pt})=H^{*}(\mathrm{pt})=\mathbb{Z}, \quad K_{c}^{*}(\mathrm{pt})=\mathbb{Z}[t]$.

\section{Chern Classes}

Let $p: L \rightarrow X$ be a line bundle over $X \in \mathbf{S m}(F)$. We define the first Chern class of $L$ in an oriented cohomology theory $A^{*}$ over $F$ by

$$
c_{1}^{A}(L)=s^{A} s_{A}\left(1_{X}\right) \in A^{1}(X),
$$

where $s: X \rightarrow L$ is the zero section of $p$ (see [7]). Since $p \circ t=\mathrm{id}_{X}$ for every section $t$ of $p$, we have $t^{A}=\left(p^{A}\right)^{-1}$ (property (iv)). Hence,

$$
c_{1}^{A}(L)=\left(p^{A}\right)^{-1} s_{A}\left(1_{X}\right) .
$$

Example 3.1. The first Chern class of a vector bundle $E \rightarrow X$ in $K$-theory is defined by

$$
c_{1}^{K}(E)=\left(\operatorname{rank}(E)-\left[E^{\vee}\right]\right) t^{-1} \in K_{0}(X) t^{-1}=K^{1}(X) .
$$

Proposition 3.2. Let $p: L \rightarrow X$ be a line bundle over $X \in \operatorname{Sm}(F)$ and let $i: Y \hookrightarrow X$ be the zero subscheme of a section $t$ of $p$. If $Y$ is a smooth divisor in $X$, then $i_{A}\left(1_{Y}\right)=c_{1}^{A}(L) \in A^{1}(X)$.

Proof. The diagram

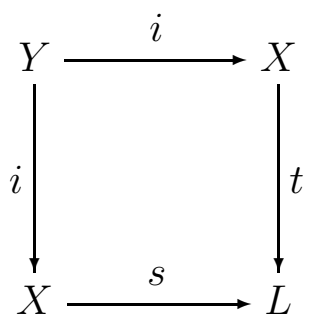

where $s$ is the zero section of $p$, is transverse. Hence,

$$
i_{A}\left(1_{Y}\right)=i_{A} i^{A}\left(1_{X}\right)=t^{A} s_{A}\left(1_{X}\right)=\left(p^{A}\right)^{-1} s_{A}\left(1_{X}\right)=c_{1}^{A}(L) .
$$

The standard method by Grothendieck (see [7]) gives Chern classes $c_{i}^{A}(E) \in$ $A^{i}(X)$ for every vector bundle $p: E \rightarrow X$ of rank $r$. They satisfy the equation

$$
\sum_{i=0}^{r}(-1)^{i} p^{A}\left(c_{i}^{A}(E)\right) \xi^{r-i}=0 \in A^{r}(\mathbb{P}(E)),
$$

where $\xi$ is the first Chern class of the tautological line bundle over $\mathbb{P}(E)$.

A partition $\alpha=\left(\alpha_{1}, \alpha_{2}, \ldots, \alpha_{k}\right)$ is a sequence of integers (possibly empty) $\alpha_{1} \geq \alpha_{2} \geq \cdots \geq \alpha_{k}>0$. The degree of $\alpha$ is the integer

$$
|\alpha|=\alpha_{1}+\alpha_{2}+\cdots+\alpha_{k} .
$$

The integer $k$ is called the length $l(\alpha)$ of the partition $\alpha$. Denote by $p(d)$ the number of all partitions of degree $d$. 
Consider the polynomial ring $\mathbb{Z}\left[b_{1}, b_{2}, \ldots\right]=\mathbb{Z}[\mathbf{b}]$ in infinitely many variables $b_{1}, b_{2}, \ldots$ as a graded ring with $\operatorname{deg} b_{i}=i$. For every partition $\alpha$ set

$$
b_{\alpha}=b_{\alpha_{1}} b_{\alpha_{2}} \ldots b_{\alpha_{k}} \text {. }
$$

The monomials $b_{\alpha}$ form a basis of the polynomial ring over $\mathbb{Z}$, and more precisely, the $b_{\alpha}$ with $|\alpha|=d$ form a basis of the $d$-graded component $\mathbb{Z}[\mathbf{b}]_{d}$. Thus, $\mathbb{Z}[\mathbf{b}]_{d}$ is a free abelian group of rank $p(d)$.

Consider another polynomial ring $\mathbb{Z}\left[c_{1}, c_{2}, \ldots\right]=\mathbb{Z}[\mathbf{c}]$ with the similar grad$\operatorname{ing} \operatorname{deg} c_{i}=i$. The elements of $\mathbb{Z}[\mathbf{c}]$ we call the characteristic classes and the $c_{n}$ - the Chern classes.

For any partition $\alpha$ we define the "smallest" symmetric polynomial

$$
P_{\alpha}\left(x_{1}, x_{2}, \ldots\right)=\sum_{\left(i_{1}, i_{2}, \ldots, i_{k}\right)} x_{i_{1}}^{\alpha_{1}} x_{i_{2}}^{\alpha_{2}} \ldots x_{i_{k}}^{\alpha_{k}}=Q_{\alpha}\left(\sigma_{1}, \sigma_{2}, \ldots\right),
$$

containing the monomial $x_{1}^{\alpha_{1}} x_{2}^{\alpha_{2}} \ldots x_{k}^{\alpha_{k}}$, where the $\sigma_{i}$ are the standard symmetric functions, and set

$$
c_{\alpha}=Q_{\alpha}\left(c_{1}, c_{2}, \ldots\right) .
$$

For example, $c_{n}=c_{(1,1, \ldots, 1)}(n$ units $)$. The characteristic classes $c_{\alpha}$ with $|\alpha|=d$ form a basis of $\mathbb{Z}[\mathbf{c}]_{d}$.

Let $A^{*}$ be an oriented cohomology theory over a field $F$. For every element (characteristic class) $c \in \mathbb{Z}[\mathbf{c}]$ and every vector bundle $E$ over a variety $X \in$ $\operatorname{Sm}(F)$ there is a well defined class $c^{A}(E) \in A^{*}(X)$. In particular, for every partition $\alpha$ there are generalized Chern classes

$$
c_{\alpha}^{A}(E) \in A^{|\alpha|}(X) .
$$

We define the characteristic polynomial of $E$ in the theory $A^{*}$ by the formula

$$
\mathbf{P}^{A}(E)=\sum_{\alpha} c_{\alpha}^{A}(E) b_{\alpha} \in A^{*}(X)[\mathbf{b}] .
$$

Example 3.3. If $L$ is a line bundle, then $\mathbf{P}^{A}(L)=\sum_{i \geq 0} c_{1}^{A}(L)^{i} b_{i}$.

Assume that a vector bundle $E \rightarrow X$ has a filtration with line factors $L_{1}, L_{2}, \ldots, L_{r}$. Then it follows from definition of the generalized Chern classes that

$$
\mathbf{P}^{A}(E)=\mathbf{P}^{A}\left(L_{1}\right) \cdot \mathbf{P}^{A}\left(L_{2}\right) \cdot \ldots \cdot \mathbf{P}^{A}\left(L_{r}\right) .
$$

Hence, by the splitting principle, for an exact sequence of vector bundles $0 \rightarrow E^{\prime} \rightarrow E \rightarrow E^{\prime \prime} \rightarrow 0$ over $X$

$$
\mathbf{P}^{A}(E)=\mathbf{P}^{A}\left(E^{\prime}\right) \cdot \mathbf{P}^{A}\left(E^{\prime \prime}\right) .
$$

The value of the $i$-th Chern class $c_{i}(E)$ is nilpotent for $i>0$ (see [7]), hence for every $\alpha \neq \emptyset$, the class $c_{\alpha}^{A}(E)$ is also nilpotent. The constant term of the polynomial $\mathbf{P}^{A}(E)$ is equal to 1 , so that the polynomial $\mathbf{P}^{A}(E)$ is invertible in the polynomial ring $A^{*}(X)[\mathbf{b}]$. Thus, there is well defined group homomorphism

$$
\mathbf{P}^{A}: K_{0}(X) \longrightarrow A^{*}(X)[\mathbf{b}]^{\times}, \quad[E] \mapsto \mathbf{P}^{A}(E)
$$


For a variety $X \in \mathbf{S m}(F)$ we define the characteristic polynomial of $X$ in the theory $A^{*}$ :

$$
\mathbf{P}_{X}^{A}=\mathbf{P}^{A}\left(T_{X}\right)^{-1}=\mathbf{P}^{A}\left(-T_{X}\right) \in A^{*}(X)[\mathbf{b}],
$$

where $T_{X}$ is the tangent bundle of $X$.

Assume that $X$ is projective. Let $p: X \rightarrow$ pt be the structure morphism. The polynomial

$$
\mathbf{F}_{X}^{A}=p_{A} \mathbf{P}_{X}^{A}=\sum_{\alpha} p_{A} c_{\alpha}\left(-T_{X}\right) b_{\alpha} \in A^{*}(\mathrm{pt})[\mathbf{b}]
$$

is called the the fundamental polynomial of $X$ in the theory $A^{*}$. The coefficients of the polynomial $\mathbf{F}_{X}^{A}$, the elements $p_{A} c_{\alpha}\left(-T_{X}\right) \in A^{*}(\mathrm{pt})$, are called the characteristic numbers of $X$ in the theory $A^{*}$. Clearly, the fundamental class $[X]^{A}$ is the constant term of the fundamental polynomial $\mathbf{F}_{X}^{A}$.

Example 3.4. Let $X \in \mathbf{S m}(F)$ be a variety of dimension $d$. Then the polynomial $\mathbf{F}_{X}^{H} \in \mathbb{Z}[\mathbf{b}]$ is either zero or homogeneous of degree $d$. The class of the tangent bundle of the projective space $\mathbb{P}_{F}^{n}$ is equal to $\left[L_{c a n}\right]^{n+1}-1$, where $L_{\text {can }}$ is the canonical bundle over $\mathbb{P}_{F}^{n}$. Since $c_{1}^{H}\left(L_{c a n}\right)^{n}$ is the class of a rational point, the polynomial $\mathbf{F}_{\mathbb{P}^{n}}^{H}$ is equal to the degree $n$ part of the power series

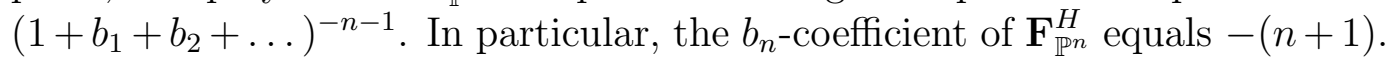
For example,

$$
\begin{aligned}
& \mathbf{F}_{\mathbb{P}^{1}}^{H}=-2 b_{1}, \\
& \mathbf{F}_{\mathbb{P}^{2}}^{H}=-3 b_{2}+6 b_{1}^{2} .
\end{aligned}
$$

Example 3.5. Note that for every vector bundle $E$ over a variety $X \in \operatorname{Sm}(F)$,

$$
c_{\alpha}^{K}(E) \in K_{0}(X)^{(|\alpha|)} t^{-|\alpha|},
$$

where $K_{0}(X)^{(i)}$ is the $i$-th term of the topological filtration on $K_{0}(X)$. Therefore, $c_{\alpha}^{K}(E)=0$ if $|\alpha|>d=\operatorname{dim} X$ (cf. Corollary 9.6). Hence, $\mathbf{F}_{X}^{K} \in \mathbb{Z}[t, \mathbf{b}]$ is a homogeneous polynomial (with $t$ of degree 1 ). The $t^{d}$-coefficient of $\mathbf{F}_{X}^{K}$ is the Todd number of $X$. For example,

$$
\begin{aligned}
& \mathbf{F}_{\mathbb{P}^{1}}^{K}=t-2 b_{1}, \\
& \mathbf{F}_{\mathbb{P}^{2}}^{K}=t^{2}-3 t b_{1}-3 b_{2}+6 b_{1}^{2} .
\end{aligned}
$$

We will prove (Proposition 6.1) that $\left.\mathbf{F}_{X}^{K}\right|_{t=0}=\mathbf{F}_{X}^{H}$ for every $X \in \mathbf{S m}(F)$.

Thus, every projective variety $X \in \mathbf{S m}(F)$ has the class $\mathbf{F}_{X}^{H}$ in $\mathbb{Z}[\mathbf{b}]$. Clearly, the class does not change under field extensions: for every field extension $E / F$ the varieties $X$ and $X_{E}=X \times_{\operatorname{Spec} F} \operatorname{Spec} E$ have the same class in Laz. Hence, if $X$ and $Y$ are twisted forms of each other (if they are isomorphic over a separable closure of $F$ ), then $\mathbf{F}_{X}^{H}=\mathbf{F}_{Y}^{H}$.

For a variety $X \in \mathbf{S m}(F)$ denote by $n_{X}$ the $\operatorname{gcd}$ of $\operatorname{deg}(x)=[F(x): F]$ over all closed points $x \in X$. By the very definition, for a projective variety $X$, all the coefficients of $\mathbf{F}_{X}^{H}$ (the characteristic numbers) are divisible by $n_{X}$. We have proved 
Proposition 3.6. 1. For every projective variety $X \in \operatorname{Sm}(F)$, the polynomial $\mathbf{F}_{X}^{H}$ is divisible by $n_{Y}$ for every twisted form $Y$ of $X_{E}$ over a field extension $E / F$.

2. Let $n$ be the gcd of all the coefficients of $\mathbf{F}_{X}^{H}$ for a projective variety $X \in$ $\operatorname{Sm}(F)$. Then $X$ has a zero-cycle of degree $n$.

Example 3.7. For every $d \in \mathbb{N}$ and a field $F$ there is a field extension $E / F$ and a division algebra $A$ over $E$ of dimension $(d+1)^{2}$. Let $Y$ be the SeveriBrauer variety over $E$ corresponding to $A$ (see [4]). The variety $Y$ if a twisted form of the projective space $\mathbb{P}_{F}^{d}$. Since $n_{Y}=d+1$, the Proposition explains why the characteristic polynomial of the projective space $\mathbb{P}_{F}^{d}$ in $\mathbb{Z}[\mathbf{b}]$ is divisible by $n+1$.

\section{TILDE OPERATION}

Let $A^{*}$ be an oriented cohomology theory over $F$. We associate to $A^{*}$ a new cohomology theory $\widetilde{A}^{*}$ defined by

$$
\widetilde{A}^{*}(X)=A^{*}(X) \otimes \mathbb{Z}[\mathbf{b}]=A^{*}(X)[\mathbf{b}] .
$$

The structure of a graded ring on $\widetilde{A}^{*}(X)$ is given by the one of the graded ring $A^{*}(X)$ and by assigning degree $-|\alpha|$ to every $b_{\alpha}$. In particular, for every $X \in \mathbf{S m}(F), \mathbf{P}_{X}^{A} \in \widetilde{A}^{0}(X)$ and, if $X$ is projective, $\mathbf{F}_{X}^{A} \in \widetilde{A}^{-d}(\mathrm{pt})$, where $d=\operatorname{dim}(X)$.

The pull-back homomorphism $f^{\widetilde{A}}: \widetilde{A}^{*}(X) \rightarrow \widetilde{A}^{*}(Y)$ associated to a morphism $f: Y \rightarrow X$ is equal to $f^{A} \otimes \mathrm{id}_{\mathbb{Z}[\mathbf{b}]}$. The push-forward map $f_{\widetilde{A}}$ associated to a projective morphism $f: Y \rightarrow X$ is defined by

$$
f_{\widetilde{A}}(a)=f_{A}\left(a \cdot \mathbf{P}_{Y}^{A}\right) \cdot\left(\mathbf{P}_{X}^{A}\right)^{-1}=f_{A}\left(a \cdot \mathbf{P}_{Y}^{A} \cdot f^{A}\left(\mathbf{P}_{X}^{A}\right)^{-1}\right) .
$$

If $f$ is a closed embedding, then $\left[f^{*} T_{X}\right]-\left[T_{Y}\right]$ is equal to the class of the normal bundle $N_{Y} X$ of $Y$ in $X$. Hence $\mathbf{P}_{Y}^{A} \cdot f^{A}\left(\mathbf{P}_{X}^{A}\right)^{-1}=\mathbf{P}^{A}\left(N_{Y} X\right)$ and

$$
f_{\widetilde{A}}(a)=f_{A}\left(a \cdot \mathbf{P}^{A}\left(N_{Y} X\right)\right) .
$$

Lemma 4.1. Let $p: L \rightarrow X$ be a line bundle. Then

$$
c_{1}^{\widetilde{A}}(L)=c_{1}^{A}(L) \cdot \mathbf{P}^{A}(L)=\sum_{i \geq 0} c_{1}^{A}(L)^{i+1} b_{i} \in A^{*}(X)[\mathbf{b}] .
$$

Proof. Let $s: X \rightarrow L$ be the zero section. The normal bundle of $s$ is equal to L. Hence,

$$
\begin{aligned}
c_{1}^{\widetilde{A}}(L) & =s^{\widetilde{A}} s_{\widetilde{A}}\left(1_{X}\right) \\
& =s^{A} s_{A}\left(\mathbf{P}^{A}(L)\right) \quad \text { (projection formula) } \\
& =s^{A}\left(s_{A}(1) \cdot p^{A} \mathbf{P}^{A}(L)\right) \\
& =c_{1}^{A}(L) \cdot \mathbf{P}^{A}(L) .
\end{aligned}
$$


Proposition 4.2. The functor $\widetilde{A}^{*}$ is an oriented cohomology theory.

Proof. We need to check properties (i)-(v) in the definition of an oriented cohomology theory.

(i) Let $f: Y \rightarrow X$ and $g: Z \rightarrow Y$ be two projective morphisms. Then for any $a \in A^{*}(Z)$,

$$
\begin{aligned}
\left(f_{\widetilde{A}} \circ g_{\widetilde{A}}\right)(a) & =f_{\widetilde{A}}\left(g_{A}\left(a \mathbf{P}_{Z}^{A}\right) \cdot\left(\mathbf{P}_{Y}^{A}\right)^{-1}\right) \\
& \left.=f_{A}\left(g_{A}\left(a \mathbf{P}_{Z}^{A}\right) \cdot\left(\mathbf{P}_{Y}^{A}\right)^{-1} \cdot \mathbf{P}_{Y}^{A}\right)\right) \cdot\left(\mathbf{P}_{X}^{A}\right)^{-1} \\
& =\left(f_{A} \circ g_{A}\right)\left(a \mathbf{P}_{Z}^{A}\right) \cdot\left(\mathbf{P}_{X}^{A}\right)^{-1} \\
& =(f \circ g)_{\widetilde{A}}(a) .
\end{aligned}
$$

(ii) Follows from Lemma 4.1 and invertibility of $\mathbf{P}^{A}(L)$.

(iii) Consider a transverse Cartesian square (1). We have

$$
\mathbf{P}_{Y^{\prime}}^{A} \cdot f^{\prime} A\left(\mathbf{P}_{Y^{\prime}}^{A}\right)^{-1}=h^{A} \mathbf{P}_{Y}^{A} \cdot h^{A} f^{A}\left(\mathbf{P}_{X}^{A}\right)^{-1}
$$

and therefore, for every $a \in A^{*}(Y)$,

$$
\begin{aligned}
\left(f_{\widetilde{A}}^{\prime} \circ h^{\widetilde{A}}\right)(a) & =f_{A}^{\prime}\left(h^{A}(a) \cdot \mathbf{P}_{Y^{\prime}}^{A} \cdot f^{\prime}\left(\mathbf{P}_{Y^{\prime}}^{A}\right)^{-1}\right) \\
& =f_{A}^{\prime}\left(h^{A}(a) \cdot h^{A} \mathbf{P}_{Y}^{A} \cdot h^{A} f^{A}\left(\mathbf{P}_{X}^{A}\right)^{-1}\right) \\
& =f_{A}^{\prime} h^{A}\left(a \cdot \mathbf{P}_{Y}^{A} \cdot f^{A}\left(\mathbf{P}_{X}^{A}\right)^{-1}\right) \\
& =g^{A} f_{A}\left(a \cdot \mathbf{P}_{Y}^{A} \cdot f^{A}\left(\mathbf{P}_{X}^{A}\right)^{-1}\right) \\
& =g^{A}\left(f_{A}\left(a \cdot \mathbf{P}_{Y}^{A}\right) \cdot\left(\mathbf{P}_{X}^{A}\right)^{-1}\right) \\
& =\left(g^{\widetilde{A}} \circ f_{\widetilde{A}}\right)(a) .
\end{aligned}
$$

(iv) Obvious.

(v) Let $f: Y \rightarrow X$ be a projective morphism in $\operatorname{Sm}(F), a \in A^{*}(X)$ and $b \in A^{*}(Y)$. We have

$$
\begin{aligned}
f_{\widetilde{A}}\left(b \cdot f^{\widetilde{A}}(a)\right) & =f_{A}\left(b \cdot f^{A}(a) \cdot \mathbf{P}_{Y}^{A}\right) \cdot\left(\mathbf{P}_{X}^{A}\right)^{-1} \quad \text { (projection formula) } \\
& =f_{A}\left(b \cdot \mathbf{P}_{Y}^{A}\right) \cdot a \cdot\left(\mathbf{P}_{X}^{A}\right)^{-1} \\
& =f_{\widetilde{A}}(b) \cdot a
\end{aligned}
$$

Remark 4.3. The correspondence $E \mapsto \mathbf{P}^{A}(E)$ is given by the characteristic class $\mathbf{P}^{A}=\sum_{\alpha} c_{\alpha} b_{\alpha}$ over $\mathbb{Z}[\mathbf{b}]$. In view of [8], $\mathbf{P}^{A}$ is the inverse Todd genus of the natural embedding of $A^{*}$ into $\widetilde{A}^{*}$ and the formula (3) is the Riemann-Roch theorem for this embedding.

Note that if $X \in \operatorname{Sm}(F)$ is projective, the fundamental class $[X]^{\widetilde{A}} \in \widetilde{A}(\mathrm{pt})=$ $A(\mathrm{pt})[\mathbf{b}]$ coincides with the fundamental polynomial $\mathbf{F}_{X}^{A}$. In particular, by Proposition 2.3, $\mathbf{F}_{X \times Y}^{A}=\mathbf{F}_{X}^{A} \cdot \mathbf{F}_{Y}^{A}$ for all $X, Y \in \mathbf{S m}(F)$. 


\section{Formal GRoup LAW OF A THEORY}

Let $A^{*}$ be an oriented cohomology theory over $F$. By [7], there is unique commutative formal group law

$$
\Phi^{A}=\sum_{i, j \geq 0} a_{i j}^{A} x^{i} y^{j}=x+y+\sum_{i, j \geq 1} a_{i j}^{A} x^{i} y^{j},
$$

over the coefficient ring $A^{*}(\mathrm{pt})$ with $a_{i j}^{A} \in A^{1-i-j}(\mathrm{pt})$, such that for every two line bundles $L$ and $L^{\prime}$ over a variety $X \in \operatorname{Sm}(F)$,

$$
c_{1}\left(L \otimes L^{\prime}\right)=c_{1}(L)+c_{1}\left(L^{\prime}\right)+\sum_{i, j \geq 1} a_{i j}^{A} c_{1}(L)^{i} c_{1}\left(L^{\prime}\right)^{j} \in A^{1}(X) .
$$

Example 5.1. Since $c_{1}^{H}\left(L \otimes L^{\prime}\right)=c_{1}^{H}(L)+c_{1}^{H}\left(L^{\prime}\right)$ for two line bundles $L$ and $L^{\prime}$ [2, Prop. 2.5(e)], $\Phi^{H}(x, y)=x+y$ is the additive group law. It follows from the description of the first Chern class in $K$-theory (Example 3.1 ) that $\Phi^{K}(x, y)=x+y-x y t$ (called the multiplicative group law).

In the rest of the section we prove that the coefficients of the group law $\Phi^{A}$ belong to the subring $A_{c}^{*}(\mathrm{pt}) \subset A^{*}(\mathrm{pt})$.

Lemma 5.2. Let $L_{\text {can }}$ be the canonical line bundle over the projective space $\mathbb{P}_{F}^{n}$ over $F$. Then $p_{A}\left(c_{1}^{A}\left(L_{\text {can }}\right)^{i}\right)=\left[\mathbb{P}_{F}^{n-i}\right]^{A}$ for every $i \geq 0$.

Proof. Induction on $n$. Let $p: \mathbb{P}_{F}^{n} \rightarrow$ pt the structure morphism, $j: \mathbb{P}_{F}^{n-1} \hookrightarrow$ $\mathbb{P}_{F}^{n}$ an embedding, $q=p \circ j, \xi=c_{1}^{A}\left(L_{c a n}\right)$. Then $j^{A}(\xi)=c_{1}^{A}\left(L_{c a n}^{\prime}\right)$, where $L_{\text {can }}^{\prime}=j^{*}\left(L_{\text {can }}\right)$ is the canonical vector bundle over $\mathbb{P}_{F}^{n-1}$. Proposition 3.2 gives $\xi=j_{A}\left(1_{\mathbb{P}^{n-1}}\right)$, hence, by the induction hypothesis,

$$
p_{A}\left(\xi^{i}\right)=p_{A}\left(j_{A}(1) \cdot \xi^{i-1}\right)=p_{A} j_{A}\left(j^{A}(\xi)^{i-1}\right)=q_{A}\left(c_{1}^{A}\left(L_{c a n}^{\prime}\right)^{i-1}\right)=\left[\mathbb{P}_{F}^{n-i}\right]^{A} .
$$

Lemma 5.3. (cf. [1, Prop. II.10.6]) Let $V$ be a smooth hypersurface in $\mathbb{P}_{F}^{n} \times \mathbb{P}_{F}^{m}$ of type $(1,1)$ for some $n$ and $m$. Then

$$
[V]^{A}=\sum_{i=0}^{n} \sum_{j=0}^{m} a_{i j}^{A}\left[\mathbb{P}_{F}^{n-i}\right]^{A} \cdot\left[\mathbb{P}_{F}^{m-j}\right]^{A} \in A^{1-n-m}(\mathrm{pt}) .
$$

Proof. Let $i: V \hookrightarrow \mathbb{P}_{F}^{n} \times \mathbb{P}_{F}^{m}$ be the embedding of $V$ as a divisor. The corresponding line bundle is the tensor product $L_{1} \otimes L_{2}$ of two canonical line bundles on $\mathbb{P}_{F}^{n}$ and $\mathbb{P}_{F}^{m}$ respectively. Hence, by Proposition 3.2,

$$
i_{A}\left(1_{V}\right)=c_{1}^{A}\left(L_{1} \otimes L_{2}\right)=\Phi^{A}(\xi, \eta)=\sum_{i, j \geq 0} a_{i j}^{A} \xi^{i} \eta^{j}
$$

where $\xi=c_{1}^{A}\left(L_{1}\right), \eta=c_{1}^{A}\left(L_{2}\right)$.

Let $p: \mathbb{P}_{F}^{n} \times \mathbb{P}_{F}^{m} \rightarrow$ pt be the structure morphism. By Lemma 5.2,

$$
p_{A}\left(\xi^{i}\right)=\left[\mathbb{P}_{F}^{n-i}\right]^{A}, \quad p_{A}\left(\eta^{j}\right)=\left[\mathbb{P}_{F}^{m-j}\right]^{A},
$$


hence, by projection formula, $p_{A}\left(\xi^{i} \eta^{j}\right)=\left[\mathbb{P}_{F}^{n-i}\right]^{A} \cdot\left[\mathbb{P}_{F}^{m-j}\right]^{A}$ and therefore,

$$
[V]^{A}=p_{A}\left(i_{A}\left(1_{V}\right)\right)=p_{A}\left(\sum_{i, j \geq 0} a_{i j}^{A} \xi^{i} \eta^{j}\right)=\sum_{i=0}^{n} \sum_{j=0}^{m} a_{i j}^{A}\left[\mathbb{P}_{F}^{n-i}\right]^{A} \cdot\left[\mathbb{P}_{F}^{m-j}\right]^{A}
$$

Corollary 5.4. $a_{n m}^{A} \in A_{c}^{1-n-m}(\mathrm{pt})$ for every $n$ and $m$.

Proof. We prove the statement by induction on $n+m$. By Lemma 5.3 and induction hypothesis, $a_{n m}-[V]^{A} \in A_{c}^{1-n-m}(\mathrm{pt})$, whence the result.

Corollary 5.5. Let $i(t)=\sum_{k \geq 1} b_{k}^{A} t^{k}$ be the additive inverse power series of $\Phi^{A}$, that is $\Phi_{A}(t, i(t))=0$. Then $b_{k}^{A} \in A_{c}^{1-k}(\mathrm{pt})$ for every $k \geq 1$.

\section{6. $K$-THEORY VERSUS CHOW THEORY}

A relation between $K$-theory and (rational) Chow-theory is given by the Chern character

$$
\operatorname{ch}_{X}: K^{*}(X) \longrightarrow H^{*}(X) \otimes \mathbb{Q}
$$

for every $X \in \mathbf{S m}(F)$. It is the ring homomorphism defined by

$$
\operatorname{ch}\left([E] t^{-k}\right)=\operatorname{ch}([E])=\operatorname{rank}(E)+\sum_{i=1}^{\infty} \frac{1}{i !} c_{(i)}^{H}(E)
$$

for a vector bundle $E \rightarrow X$ [2, Ch. 15]. In particular, the homomorphism

$$
\operatorname{ch}_{\mathrm{pt}}: K^{*}(\mathrm{pt})=\mathbb{Z}\left[t, t^{-1}\right] \longrightarrow \mathbb{Q}=H^{*}(\mathrm{pt}) \otimes \mathbb{Q}
$$

is the evaluation at $t=1$.

For a projective morphism $f: X \rightarrow Y$, by the classical Riemann-Roch formula [2, Th. 15.2], for every $a \in K^{*}(X)$ :

$$
f_{H}\left(\operatorname{ch}_{X}(a) \cdot t d\left(T_{X}\right)\right)=\operatorname{ch}_{Y}\left(f_{K}(a)\right) \cdot t d\left(T_{Y}\right),
$$

where $t d \in \mathbb{Q}[\mathbf{c}]$ is the (rational) Todd characteristic class.

Let $X \in \operatorname{Sm}(F)$ be a projective variety of dimension $d$ and $p: X \rightarrow$ pt the structure morphism. Applying the Riemann-Roch formula for $p$ and $a=$ $c_{\alpha}^{K}\left(-T_{X}\right)$, we get

(4) $\operatorname{deg}\left(\operatorname{ch}_{X}\left(c_{\alpha}^{K}\left(-T_{X}\right)\right) \cdot t d\left(T_{X}\right)\right)=\operatorname{ch}_{\mathrm{pt}}\left(p_{K}\left(c_{\alpha}^{K}\left(-T_{X}\right)\right)\right)=\left.p_{K} c_{\alpha}^{K}\left(-T_{X}\right)\right|_{t=1}$,

where $\operatorname{deg}=p_{H}$ is the degree homomorphism.

Proposition 6.1. 1. For every projective $X \in \mathbf{S m}(F),\left.\mathbf{F}_{X}^{K}\right|_{t=0}=\mathbf{F}_{X}^{H}$.

2. The evaluation homomorphism $\mathbb{Z}[t, \mathbf{b}] \rightarrow \mathbb{Z}[\mathbf{b}]$ at $t=0$ induces ring isomorphism between $\widetilde{K}_{c}(\mathrm{pt})$ and $\widetilde{H}_{c}(\mathrm{pt})$. In particular, for every $d$, the degree $d$ components $\widetilde{K}_{c}(\mathrm{pt})_{d}$ and $\widetilde{H}_{c}(\mathrm{pt})_{d}$ are free abelian groups of rank at most $p(d)$. 
Proof. 1. Let $d=\operatorname{dim} X$. For every partition $\alpha$,

$$
p_{K} c_{\alpha}^{K}\left(-T_{X}\right) \in K^{|\alpha|-d}(X)=K_{0}(X) t^{d-|\alpha|} .
$$

a) If $|\alpha|>d$, then $c_{\alpha}^{K}\left(-T_{X}\right)$ and $c_{\alpha}^{H}\left(-T_{X}\right)$ are both zero (Examples 3.4 and $3.5)$.

b) If $|\alpha|<d$, then $\left.p_{K} c_{\alpha}^{K}\left(-T_{X}\right)\right|_{t=0}=0=\operatorname{deg} c_{\alpha}^{H}\left(-T_{X}\right)$.

c) Assume that $|\alpha|=d$. We have

$$
\operatorname{ch}\left(c_{\alpha}^{K}\right) \cdot t d^{-1}=c_{\alpha}^{H}+\text { characteristic class of degree }>d .
$$

Hence, by (4),

$$
\left.p_{K} c_{\alpha}^{K}\left(-T_{X}\right)\right|_{t=0}=p_{K} c_{\alpha}^{K}\left(-T_{X}\right)=\left.p_{K} c_{\alpha}^{K}\left(-T_{X}\right)\right|_{t=1}=\operatorname{deg} c_{\alpha}^{H}\left(-T_{X}\right) .
$$

2. By the first statement, the evaluation at $t=0$ takes $\widetilde{K}_{c}(\mathrm{pt})$ onto $\widetilde{H}_{c}(\mathrm{pt})$. We need to prove injectivity of the evaluation. Let $X_{1}, \ldots, X_{s} \in \mathbf{S m}(F)$ be projective varieties of the same dimension and $m_{1}, \ldots, m_{s} \in \mathbb{Z}$ such that

$$
\left.\sum_{i=1}^{s} m_{i} \mathbf{F}_{X_{i}}^{K}\right|_{t=0}=\sum_{i=1}^{s} m_{i} \mathbf{F}_{X_{i}}^{H}=0
$$

Equivalently,

$$
\sum_{i=1}^{s} m_{i} \operatorname{deg} c^{H}\left(-T_{X_{i}}\right)=0
$$

for every generalized Chern class $c=c_{\alpha}$. Since $c_{\alpha}$ generate $\mathbb{Q}[\mathbf{c}]$, the formula (5) holds for every characteristic class $c \in \mathbb{Q}[\mathbf{c}]$. Taking $c=\operatorname{ch}\left(c_{\alpha}\right) \cdot t d^{-1}$ and applying formula (4) for every $X_{i}$, we get

$$
\left.\sum_{i=1}^{s} m_{i} p_{K}^{(i)} c_{\alpha}^{K}\left(-T_{X_{i}}\right)\right|_{t=1}=0
$$

for every $\alpha$, where $p^{(i)}: X_{i} \rightarrow$ pt is the structure morphism. But the sum $\sum_{i=1}^{s} m_{i} p_{K}^{(i)} c_{\alpha}^{K}\left(-T_{X_{i}}\right)$ is a monomial in $t$ and hence it is zero for every $\alpha$. It follows that $\sum_{i=1}^{s} m_{i} \mathbf{F}_{X_{i}}^{K}=0$.

The group $\widetilde{H}_{c}(\mathrm{pt})_{d}$ is a subgroup of the free group $\mathbb{Z}[\mathbf{b}]_{d}$ of rank $p(d)$, whence the last statement of the Proposition.

\section{Hypersurfaces $V\left(n_{1}, n_{2}, \ldots, n_{k}\right)$}

¿From now on we assume that the base field is infinite. We will use the following variant of Bertini theorem [3, Th.II.8.18]:

Theorem 7.1. Let $X$ be a smooth variety over an infinite field, $L$ a very ample line bundle over $X$. Then there is a section of $L$ with smooth zero divisor.

Let $\mathbb{P}$ be the product of projective spaces $\mathbb{P}_{F}^{n_{1}} \times \mathbb{P}_{F}^{n_{2}} \times \cdots \times \mathbb{P}_{F}^{n_{k}}$. Denote by $L_{i}$ the pull-back on $\mathbb{P}$ of the canonical vector bundle over $\mathbb{P}_{F}^{n_{i}}$ and by $L$ the tensor product of the $L_{i}$. By Theorem 7.1, there is a smooth hypersurface

$$
V=V\left(n_{1}, n_{2}, \ldots, n_{k}\right) \subset \mathbb{P},
$$


which is the zero divisor of a section of $L$.

Let $i: V \hookrightarrow \mathbb{P}$ be the embedding. For an oriented cohomology theory $A^{*}$ over $F$, by Proposition 3.2,

$$
i_{\widetilde{A}}\left(1_{V}\right)=c_{1}^{\widetilde{A}}(L)=c_{1}^{A}(L) \cdot \mathbf{P}^{A}(L)
$$

Denote by $q: \mathbb{P} \rightarrow$ pt the structure morphism. Hence,

$$
\mathbf{F}_{V}^{A}=[V]^{\widetilde{A}}=q_{\widetilde{A}} i_{\widetilde{A}}\left(1_{V}\right)=q_{\widetilde{A}}\left(c_{1}^{A}(L) \cdot \mathbf{P}^{A}(L)\right)=q_{A}\left(c_{1}^{A}(L) \cdot \mathbf{P}^{A}(L) \cdot \mathbf{P}_{\mathbb{P}}^{A}\right) .
$$

The class in $K_{0}(\mathbb{P})$ of the tangent bundle of $\mathbb{P}$ equals $\sum\left[L_{i}\right]^{n_{i}+1}-k 1$. We have then

$$
\mathbf{P}_{\mathbb{P}}^{A}=\prod_{i=1}^{k} \mathbf{P}^{A}\left(L_{i}\right)^{-n_{i}-1}
$$

Thus,

$$
\mathbf{F}_{V}^{A}=q_{A}\left(c_{1}^{A}(L) \cdot \mathbf{P}^{A}(L) \cdot \prod_{i=1}^{k} \mathbf{P}^{A}\left(L_{i}\right)^{-n_{i}-1}\right) .
$$

Set $\xi_{i}=c_{1}^{A}\left(L_{i}\right), \xi=c_{1}^{A}(L)$. Therefore,

$$
\mathbf{F}_{V}^{A}=q_{A}\left(\left(\sum_{j \geq 0} \xi^{j+1} b_{j}\right) \cdot \prod_{i=1}^{k}\left(\sum_{j \geq 0} \xi_{i}^{j} b_{j}\right)^{-n_{i}-1}\right)
$$

Note that

$$
\xi=\Psi^{A}\left(\xi_{1}, \xi_{2}, \ldots, \xi_{k}\right),
$$

where $\Psi^{A}$ is the iterated group law of $A$.

Assume that $A^{*}=H^{*}$, so that $\xi=\sum \xi_{i}$. We would like to compute the $\alpha$-characteristic number of $V$ for $\alpha=(n-1)$, where $n=\sum n_{i}$, that is the coefficient of $b_{n-1}$ in $\mathbf{F}_{V}^{H}$. Assume that $n_{i}>1$ for at least two values of $i$, so that $n-1 \geq n_{i}+1$ for all $i$. Since $\xi_{i}^{n_{i}+1}=0$, we can ignore the second multiple in (6). Hence

$$
\operatorname{deg} c_{(n-1)}^{H}\left(-T_{V}\right)=q_{A}\left(\xi^{n}\right)=\frac{n !}{n_{1} ! n_{2} ! \ldots n_{k} !} q_{A}\left(\xi_{1}^{n_{1}} \ldots \xi_{k}^{n_{k}}\right)=\frac{n !}{n_{1} ! n_{2} ! \ldots n_{k} !} .
$$

We have proved

Proposition 7.2. (cf. [9, Lemma VII.6.8], [10]) Let $V=V\left(n_{1}, n_{2}, \ldots, n_{k}\right)$, $n=\sum n_{i}$. If $n_{i}>1$ for at least two values of $i$, then

$$
\operatorname{deg} c_{(n-1)}^{H}(V)=\frac{n !}{n_{1} ! n_{2} ! \ldots n_{k} !} .
$$

Now consider $K$-theory $A^{*}=K^{*}$. Let $p$ be a prime integer. Assume that for some $s, n_{i}=p^{s-1}$ for every $i$ and $k=p$, so that $n=p^{s}$. We have

$$
\xi=\Phi\left(\xi_{1}, \xi_{2}, \ldots, \xi_{p}\right)=v_{1}-v_{2} t+\cdots+(-1)^{p} v_{p} t^{p-1},
$$

where $v_{j}$ are the standard symmetric functions on the $\xi_{i}$. Note that the r.h.s. of (6) is a polynomial in the $b_{\alpha}$ with the coefficients of the form $q_{K}(P(v))$, where $P$ is a polynomial over $\mathbb{Z}$. 
Lemma 7.3. Let $v$ be a monomial $v_{1}^{\alpha_{1}} \ldots v_{p}^{\alpha_{p}}$. If $\alpha_{i}>0$ for some $i=$ $1,2, \ldots, p-1$, then $q_{K}(v)$ is divisible by $p$.

Proof. Assume $\alpha_{i}>0$, so that $v=v_{i} u$, where $u=v_{i}^{-1} v$ is a monomial. For every permutation $\sigma \in S_{p}$ let $\xi_{\sigma}=\xi_{\sigma(1)} \xi_{\sigma(2)} \ldots \xi_{\sigma(i)}$. Then $v=\sum_{\sigma \in S_{p}} \xi_{\sigma} u$ and hence $q_{K}(v)$ is divisible by $p$ since $q_{K}\left(\xi_{\sigma} u\right)=q_{K}\left(\xi_{\tau} u\right)$ for every $\sigma, \tau \in S_{p}$ and the number $\left(\begin{array}{l}p \\ i\end{array}\right)$ of terms in the sum is divisible by $p$.

Thus, we can delete all monomials in the $v_{i}$ 's containing $v_{i}$ for $i=1,2, \ldots$, $p-1$ and rewrite (6) modulo $p$ :

$$
\mathbf{F}_{V}^{K} \equiv q_{K}\left(\left(\sum_{j \geq 0}\left[(-1)^{p} v_{p} t^{p-1}\right]^{j+1} b_{j}\right) \cdot \prod_{i=1}^{p}\left(\sum_{j \geq 0} \xi_{i}^{j} b_{j}\right)^{-p^{s-1}-1}\right) \quad(\bmod p) .
$$

Recall that $\mathbf{F}_{V}^{K}$ is a homogeneous polynomial in $\mathbb{Z}[t, \mathbf{b}]$ of degree $\operatorname{dim}(V)=$ $p^{s}-1$.

Proposition 7.4. (cf. [10, Lemma, p.121]) Let $V=V\left(p^{s-1}, p^{s-1}, \ldots, p^{s-1}\right)(p$ terms), $\alpha=\left(p^{s-1}-1, p^{s-1}-1, \ldots, p^{s-1}-1\right)$. Then the $b_{\alpha}$-coefficient of $\mathbf{F}_{V}^{K}$ is not divisible by $p$. If $\operatorname{deg} \beta \geq p^{s}-p$ and $b_{\beta}$-coefficient of $\mathbf{F}_{V}^{K}$ is not divisible by $p$, then $\operatorname{deg} \beta=p^{s}-p$ and $\beta$ is a refinement of $\alpha$.

Proof. A typical monomial of the r.h.s. of (7) is of the form

$$
t^{(p-1)(j+1)} b_{j} b_{\alpha^{1}} \ldots b_{\alpha^{p}}=t^{(p-1)(j+1)} b_{\beta}
$$

for partitions $\alpha^{1}, \ldots, \alpha^{p}$. Note that since $v_{p} \xi_{i}^{p^{s}}=0$ we may assume that $\left|\alpha^{i}\right| \leq p^{s-1}-1$ for all $i$. We have $|\beta|=p^{s}-1-(p-1)(j+1) \leq p^{s}-p$ and equality holds iff $j=0$. Hence, if $\operatorname{deg} \beta \geq p^{s}-p$ and the $b_{\beta}$-coefficient of $\mathbf{F}_{V}^{K}$ is not divisible by $p$, then $\operatorname{deg} \beta=p^{s}-p$ and $j=0$. Therefore, $\left|\alpha^{i}\right|=p^{s-1}-1$ for all $i$ and $\beta$ is a refinement of $\alpha$.

It follows from (7) that modulo $p$, the $b_{\alpha}$-coefficient of $\mathbf{F}_{V}^{K}$ is equal to $(-1)^{p} t^{p-1} q_{K}\left(v_{p}^{p^{s-1}}\right)=(-1)^{p} t^{p-1}$ and hence it is not trivial.

Define the following partial ordering on the set of all partitions. We write $\alpha \leq \beta$ if $|\alpha|<|\beta|$ or $|\alpha|=|\beta|$ and $l(\alpha) \geq l(\beta)$. We consider largest monomials of polynomials in the $b_{i}^{\prime} \mathrm{s}$ with respect to this ordering.

Lemma 7.5. (cf. [10, Proposition, p.125]) For every prime integer $p$ and every integer $d \geq 1$ there exists a projective variety $M_{d}^{p} \in \operatorname{Sm}(F)$ of dimension $d$ such that the polynomial $\mathbf{F}_{M_{d}^{p}}^{K}$ has largest monomial $b_{d}$ modulo $p$ if $d \neq p^{s}-1$ for any $s$ or $t^{p-1}\left(b_{p^{s-1}-1}\right)^{p}$ if $d=p^{s}-1$ for some $s>0$.

Proof. Assume first that $d+1$ is not divisible by $p$ and set $M_{d}^{p}=\mathbb{P}_{F}^{d}$. By Proposition 6.1, the $b_{d}$-coefficients of $\mathbf{F}_{M_{d}^{p}}^{K}$ and $\mathbf{F}_{M_{d}^{p}}^{H}$ coincide. By Example 3.4, this coefficient is equal to $-(d+1)$ and it is not divisible by $p$.

Assume now that $d+1$ is divisible by $p$ but $d+1 \neq p^{s}$ for any $s$. We write $d+1=p^{r}(p u+v)$ with $r>0$ and $0<v<p$. If $u=0, v>1$, we set 
$M_{d}^{p}=V\left(p^{r}, p^{r}(v-1)\right)$. By Proposition 7.2 , the $b_{d}$-coefficient of $\mathbf{F}_{M_{d}^{p}}^{H}$ is equal to $\left(\begin{array}{c}p^{r} v \\ p^{r}\end{array}\right)$ and hence it is not divisible by $p$.

If $u>0$, let $M_{d}^{p}=V\left(p^{r} v, p^{r+1} u\right)$ and again by Proposition 7.2 , the $b_{d^{-}}$ coefficient of $\mathbf{F}_{M_{d}^{p}}^{H}$ is equal to $\left(\begin{array}{c}p^{r}(p u+v) \\ p^{r} v\end{array}\right)$ and it is not divisible by $p$.

If $d+1=p^{s}$ for some $s$, let $M_{d}^{p}=V\left(p^{s-1}, p^{s-1}, \ldots, p^{s-1}\right)$ ( $p$ terms). Then by Proposition 7.4 , the $b_{d}$-coefficient of $\mathbf{F}_{M_{d}^{p}}^{K}$ is zero modulo $p$ if $|\alpha| \geq p^{s}-p$ unless $|\alpha|=p^{s}-p$ and $\alpha$ refines $\left(p^{s-1}-1, \ldots,\left(p^{s-1}-1\right)\right.$.

Corollary 7.6. (cf. [10, Corollary, p.126]) For a partition $\alpha$ and let $M_{\alpha}^{p}=$ $M_{\alpha_{1}}^{p} \times \cdots \times M_{\alpha_{r}}^{p}$. Then for every integer $d \geq 0$, the polynomials $\mathbf{F}_{M_{\alpha}^{p}}^{K}(\bmod p)$ in $(\mathbb{Z} / p \mathbb{Z})[t, \mathbf{b}]$ with $|\alpha|=d$ are linearly independent.

Proposition 7.7. 1. The ring $\widetilde{K}_{c}(\mathrm{pt}) \otimes \mathbb{Z} / p \mathbb{Z}$ (resp. $\widetilde{H}_{c}(\mathrm{pt}) \otimes \mathbb{Z} / p \mathbb{Z}$ ) is a polynomial ring over $\mathbb{Z} / p \mathbb{Z}$ in the variables $\mathbf{F}_{M_{d}^{p}}^{K}\left(\operatorname{resp} . \mathbf{F}_{M_{d}^{p}}^{H}\right)(\bmod p), d \geq 1$.

2. The degree $d$ component $\widetilde{K}_{c}(\mathrm{pt})_{d}$ is a direct summand of $\mathbb{Z}[t, \mathbf{b}]_{d}$ of rank $p(d)$.

3. The degree $d$ component $\widetilde{H}_{c}(\mathrm{pt})_{d} \subset \mathbb{Z}[\mathbf{b}]_{d}$ is a free subgroup of (maximal) $\operatorname{rank} p(d)$.

Proof. By Corollary 7.6, the rank of the image of $\widetilde{K}_{c}(\mathrm{pt})_{d}$ in $(\mathbb{Z} / p \mathbb{Z})[t, \mathbf{b}]$ for every prime integer $p$ is at least $p(d)$. On the other hand, the rank of $\widetilde{K}_{c}(\mathrm{pt})_{d}$ is at most $p(d)$ by Proposition 6.1. Hence the classes $\mathbf{F}_{M_{\alpha}^{p}}^{K}(\bmod p)$ generate $\widetilde{K}_{c}(\mathrm{pt}) \otimes \mathbb{Z} / p \mathbb{Z}$. The map $\widetilde{K}_{c}(\mathrm{pt})_{d} \otimes \mathbb{Z} / p \mathbb{Z} \rightarrow(\mathbb{Z} / p \mathbb{Z})[t, \mathbf{b}]$ is injective for every $p$, therefore $\widetilde{K}_{c}(\mathrm{pt})_{d}$ is a direct summand of $\mathbb{Z}[t, \mathbf{b}]_{d}$. The statements about $\widetilde{H}_{c}(\mathrm{pt})$ follow from Proposition 6.1.

Remark 7.8. The second statement of the Proposition is an algebraic analog of the Hattori-Stong Theorem [10, Theorem, p.129].

Let $J$ be the ideal in $\widetilde{K}_{c}(\mathrm{pt}) \otimes \mathbb{Z} / p \mathbb{Z}$ generated by $\mathbf{F}_{X}^{K}$ for all projective $X \in \operatorname{Sm}(F)$ of positive dimension. For every projective $X \in \operatorname{Sm}(F)$ of dimension $d$,

$$
\mathbf{F}_{X}^{K} \equiv \lambda \mathbf{F}_{M_{d}^{p}}^{K}\left(\bmod J^{2}\right)
$$

for a uniquely determined $\lambda \in \mathbb{Z} / p \mathbb{Z}$. Recall that the $b_{d}$-coefficients of $\mathbf{F}_{X}^{K}$ and $\mathbf{F}_{X}^{H}$ coincide and are equal to $\operatorname{deg} c_{(d)}^{H}\left(-T_{X}\right)$. Note that the $b_{d}$-coefficient of every element of $J^{2}$ is trivial for every $d$.

Proposition 7.9. For a projective variety $X \in \operatorname{Sm}(F)$ of dimension $d=$ $p^{s}-1$, the characteristic number $\operatorname{deg} c_{(d)}^{H}\left(-T_{X}\right)$ is divisible by $p$.

Proof. The statement follows from (8) since by Lemma $7.5, \operatorname{deg} c_{(d)}^{H}\left(-T_{M_{d}^{p}}\right)$ is divisible by $p$.

Lemma 7.10. Let $S$ a set of smooth projective varieties over $F$. Assume that for every prime integer $p$ and every $d \geq 1$ there is $X \in S$ such that 
$\operatorname{deg} c_{(d)}^{H}\left(-T_{X}\right)$ is not divisible by $p$ if $d \neq p^{s}$ for any $s$ and $\operatorname{deg} c_{(d)}^{H}\left(-T_{X}\right)$ is not divisible by $p^{2}$ if $d=p^{s}-1$ for some $s>0$. Then the ring $\widetilde{K}_{c}(\mathrm{pt})$ is generated by the $\mathbf{F}_{X}^{K}, X \in S$.

Proof. Let $p$ be a prime integer. For every $d \geq 1$, there is $X \in S$ such that $\lambda$ in (8) is not zero modulo $p$. Hence the polynomials $\mathbf{F}_{X}^{K}$ generate $\widetilde{K}_{c}(\mathrm{pt})$ modulo $p$ for every $p$, whence the statement.

Proposition 7.11. The subring $\widetilde{K}_{c}(\mathrm{pt}) \subset \mathbb{Z}[t, \mathbf{b}]$ is generated by the classes of projective spaces $\mathbb{P}_{F}^{n}$ and smooth hypersurfaces $V(n, m)$.

Proof. Let $S$ be the set of all projective spaces $\mathbb{P}_{F}^{n}$ and smooth hypersurfaces $V(n, m)$. Let $p$ be a prime integer and $d \geq 1$. If $d \neq p^{s}$ for any $s$, the proof of Lemma 7.5 shows the there is $X \in S$ such that $\operatorname{deg} c_{(d)}^{H}\left(-T_{X}\right)$ is not divisible by $p$.

Assume that $d=p^{s}-1$ for some $s$. If $s>1$, then by Proposition 7.2,

$$
\operatorname{deg} c_{\left(p^{s}-1\right)}^{H}\left(V_{p^{s-1}, p^{s}-p^{s-1}}\right)=\left(\begin{array}{c}
p^{s} \\
p^{s-1}
\end{array}\right)
$$

is not divisible by $p^{2}$. If $s=1$, by Example 3.4,

$$
\operatorname{deg} c_{(p-1)}^{H}\left(\mathbb{P}^{p-1}\right)=-p .
$$

By Lemma 7.10 , the set $S$ generates $\widetilde{K}_{c}(\mathrm{pt})$.

Propositions 6.1 and 7.11 imply

Corollary 7.12. The subring $\widetilde{H}_{c}(\mathrm{pt}) \subset \mathbb{Z}[\mathbf{b}]$ is generated by the fundamental polynomials of projective spaces $\mathbb{P}_{F}^{n}$ and smooth hypersurfaces $V(n, m)$.

\section{LAZARD RING}

Let Laz be the Lazard ring, the coefficient ring of the universal (one-dimensional, commutative) group law [9, Prop. VII.5.3]. For a commutative ring $R$, the set of $R$-points

$$
\operatorname{Spec}(\operatorname{Laz})(R)=\operatorname{Mor}(\operatorname{Spec}(R), \operatorname{Spec}(\operatorname{Laz}))=\operatorname{Hom}_{\text {rings }}(\operatorname{Laz}, R)
$$

is identified with the set of all formal group laws over $R$.

Denote the scheme Spec $\mathbb{Z}[\mathbf{b}]$ by $G$. For a commutative ring $R$ the set of $R$-points $G(R)=\operatorname{Hom}_{\text {rings }}(\mathbb{Z}[\mathbf{b}], R)$ can be identified with the set of sequences $\left(r_{1}, r_{2}, \ldots\right)$ of elements of $R\left(r_{i}\right.$ being the image of the $\left.b_{i}\right)$ and therefore with the set of power series

$$
t+r_{1} t^{2}+r_{2} t^{3}+\cdots \in R[[t]]
$$

The composition of power series makes $G$ a group scheme over $\mathbb{Z}$.

The group $\operatorname{Spec}(\mathbb{Z}[\mathbf{b}])(R)$ acts on $\operatorname{Spec}(\operatorname{Laz})(R)$ by conjugation

$$
(f \cdot \Phi)(x, y)=f\left(\Phi\left(f^{-1}(x), f^{-1}(y)\right)\right) .
$$


Thus, the group scheme $G$ acts on the scheme $\operatorname{Spec}(\mathrm{Laz})$. Denote by

$$
\log t=t+m_{1} t^{2}+m_{2} t^{3}+\cdots \in \mathbb{Z}[\mathbf{b}][[t]]
$$

the formal inverse of

$$
\exp t=t+b_{1} t^{2}+b_{2} t^{3}+\cdots \in \mathbb{Z}[\mathbf{b}][[t]] .
$$

It is known that $m_{d}=\mathbf{F}_{\mathbb{P}^{d}}^{H} /(d+1)$ [9, VII, Cor. 6.12 ].

Lemma 8.1. For every oriented cohomology theory $A^{*}$,

$$
\Phi^{\widetilde{A}}(x, y)=\exp \Phi^{A}(\log x, \log y) .
$$

Proof. For a line bundle $L$,

$$
c_{1}^{\widetilde{A}}(L)=\exp c_{1}^{A}(L), \quad c_{1}^{A}(L)=\log c_{1}^{\widetilde{A}}(L) .
$$

Hence for a pair of line bundles $L$ and $L^{\prime}$,

$$
\begin{aligned}
c_{1}^{\widetilde{A}}\left(L \otimes L^{\prime}\right) & =\exp c_{1}^{A}\left(L \otimes L^{\prime}\right) \\
& =\exp \Phi^{A}\left(c_{1}^{A}(L), c_{1}^{A}\left(L^{\prime}\right)\right) \\
& =\exp \Phi^{A}\left(\log c_{1}^{\widetilde{A}}(L), \log c_{1}^{\widetilde{A}}\left(L^{\prime}\right)\right) .
\end{aligned}
$$

By Lemma 8.1, the group law

$$
\Phi=\exp (\log x+\log y)=x+y+\sum_{i, j \geq 1} a_{i j} x^{i} y^{j}
$$

over $\mathbb{Z}[\mathbf{b}]$ coincides with $\Phi^{\widetilde{H}}$. It defines a ring homomorphism Laz $\rightarrow \mathbb{Z}[\mathbf{b}]$ which is, in fact, injective $[9, \mathrm{VII}, \S 5]$. We will identify Laz with its image in $\mathbb{Z}[\mathbf{b}]$. The ring Laz is generated by the coefficients $a_{i j}$ and $\Phi$ is the universal group law over Laz.

Theorem 8.2. For every infinite field $F$, the subgroup in $\mathbb{Z}[\mathbf{b}]$, generated by the fundamental polynomials $\mathbf{F}_{X}^{H}$ for all $X \in \mathbf{S m}(F)$, coincides with Laz $\subset \mathbb{Z}[\mathbf{b}]$.

Proof. The differential form

$$
\mathrm{d} \log (x)=\left(1+2 m_{1} x+3 m_{2} x^{2}+\ldots\right) \mathrm{d} x=\left(1+\mathbf{F}_{\mathbb{P}^{1}}^{H} x+\mathbf{F}_{\mathbb{P}^{2}}^{H} x^{2}+\ldots\right) \mathrm{d} x
$$

can be computed out of the formal group law by the formula [9, Prop. VII.5.7]

$$
\mathrm{d} \log (x)=\frac{\mathrm{d} x}{\Phi_{y}(x, 0)} .
$$

Hence, the classes of the projective spaces $\mathbb{P}_{F}^{n}$ can be expressed in terms of the $a_{i j}$, so that $\mathbf{F}_{\mathbb{P}^{n}}^{H} \in$ Laz. By Lemma $5.3, \mathbf{F}_{V(n, m)}^{H} \in$ Laz for every $n$ and $m$. It follows from Corollary 7.12 that $\widetilde{H}_{c}(\mathrm{pt}) \subset$ Laz.

Conversely, the inclusion Laz $\subset \widetilde{H}_{c}(\mathrm{pt})$ follows from Corollary 5.4 since Laz is generated by the coefficients $a_{i j}$.

Thus, every projective variety $X \in \mathbf{S m}(F)$ has the class $\mathbf{F}_{X}^{H}$ in the Lazard ring Laz. 


\section{VAlues of CHARACTERISTIC Classes}

In this section we prove that characteristic classes in an oriented cohomology theory $A^{*}$ over $F$ take values in $A_{c}^{*} \subset A^{*}$. For $X \in \operatorname{Sm}(F)$ denote by $A_{c l}^{*}(X)$ the subgroup in $A_{c}^{*}(X)$ generated by the elements $i_{A}\left(1_{Z}\right)$, where $i: Z \hookrightarrow X$ is a smooth closed subvariety.

Lemma 9.1. Let $L$ be a very ample line bundle over $X \in \operatorname{Sm}(F)$. Then $c_{1}^{A}(L) \cdot A_{c l}^{*}(X) \subset A_{c l}^{*}(X)$.

Proof. Let $i: Z \hookrightarrow X$ be a smooth closed subvariety. The restriction $L^{\prime}=\left.L\right|_{Z}$ is very ample over $Z$. By Bertini theorem 7.1, there exists a section of $L^{\prime}$ with the smooth zero divisor $Z^{\prime}$. Denote by $j: Z^{\prime} \hookrightarrow Z$ the embedding. Then by Proposition $3.2, j_{A}\left(1_{Z^{\prime}}\right)=c_{1}^{A}\left(L^{\prime}\right)$ and hence

$c_{1}^{A}(L) \cdot i_{A}\left(1_{Z}\right)=i_{A}\left(i^{A} c_{1}(L)\right)=i_{A}\left(c_{1}^{A}\left(L^{\prime}\right)\right)=i_{A} j_{A}\left(1_{Z^{\prime}}\right)=(i j)_{A}\left(1_{Z^{\prime}}\right) \in A_{c l}^{*}(X)$.

Proposition 9.2. Let $L$ be a line bundle over $X \in \operatorname{Sm}(F)$. Then $c_{1}^{A}(L) \cdot A_{c}^{*}(X) \subset A_{c}^{*}(X)$.

Proof. Let $f: Y \rightarrow X$ be a projective morphism with $Y \in \operatorname{Sm}(F)$ and let $L^{\prime}=f^{*}(L)$. Choose very ample line bundles $L_{1}$ and $L_{2}$ over $Y$ such that $L^{\prime}=L_{1} \otimes L_{2}^{-1}$. By Lemma 9.1,

$$
c_{1}^{A}\left(L_{1}\right)^{i} \cdot c_{1}^{A}\left(L_{2}\right)^{j} \in A_{c l}^{*}(Y) \subset A_{c}^{*}(Y)
$$

for all $i$ and $j$. Then by Proposition 5.4 and Corollary 5.5,

$$
c_{1}^{A}\left(L^{\prime}\right)=\Phi^{A}\left(c_{1}^{A}\left(L_{1}\right), i c_{1}^{A}\left(L_{2}\right)\right) \in A_{c}^{*}(Y) .
$$

Finally,

$$
c_{1}^{A}(L) \cdot f_{A}\left(1_{Y}\right)=f_{A}\left(f^{A} c_{1}^{A}(L)\right)=f_{A}\left(c_{1}^{A}\left(L^{\prime}\right)\right) \in f_{A}\left(A_{c}^{*}(Y)\right) \subset A_{c}^{*}(X) .
$$

Let $E$ be a vector bundle of rank $r>0$ over $X$. Consider the projection $p: \mathbb{P}(E) \rightarrow X$ and set

$$
\xi=c_{1}^{A}\left(L_{\text {can }}\right) \in A^{1}(\mathbb{P}(E)),
$$

where $L_{\text {can }}$ is the canonical line bundle over $\mathbb{P}(E)$.

Lemma 9.3. For every $i \geq 0$,

$$
p_{A}\left(\xi^{r-1+i}\right)=c_{i}^{A}(-E)+\sum_{j>i} a_{j} d_{j}^{A}(E) \in A^{*}(X),
$$

for some $a_{j} \in A_{c}^{*}(\mathrm{pt})$ and characteristic classes $d_{j}$ of degree $j$.

Proof. By the splitting principle, we may assume that $E$ is a subbundle of a trivial bundle $E^{\prime}$ of rank $n$ and the factor bundle $E^{\prime} / E$ has a filtration by line bundles $L_{1}, L_{2}, \ldots$ Let $l: \mathbb{P}(E) \rightarrow \mathbb{P}\left(E^{\prime}\right)$ be the closed embedding, $q: \mathbb{P}\left(E^{\prime}\right) \rightarrow X$ the projection, $L_{c a n}^{\prime}$ the canonical line bundle over 
$\mathbb{P}\left(E^{\prime}\right), \zeta=c_{1}^{A}\left(L_{c a n}^{\prime}\right) \in A^{1}\left(\mathbb{P}\left(E^{\prime}\right)\right)$. We can consider $l$ as a composition of closed embeddings of codimension 1 corresponding to the line bundles $q^{*} L_{k} \otimes L_{c a n}^{\prime}$. Hence, by Proposition 3.2,

$$
l_{A}\left(\xi^{r-1+i}\right)=l_{A}\left(1 \cdot l^{A} \zeta^{r-1+i}\right)=\zeta^{r-1+i} \cdot \prod_{k} c_{1}^{A}\left(q^{*} L_{k} \otimes L_{c a n}^{\prime}\right) .
$$

We can compute $c_{1}^{A}\left(q^{*} L_{k} \otimes L_{\text {can }}^{\prime}\right)$ using the formal group law $\Phi^{A}$ :

$$
\left.c_{1}^{A}\left(q^{*} L_{k} \otimes L_{c a n}^{\prime}\right)\right)=q^{A} c_{1}^{A}\left(L_{k}\right)+\zeta+\sum_{l, m \geq 1} a_{l m} q^{A} c_{1}^{A}\left(L_{k}\right)^{l} \zeta^{m} .
$$

Applying $q_{A}$ to (9) and using (10), we get the formula we need, since by Lemma 5.2, $q_{A}\left(\zeta^{s}\right)=\left[\mathbb{P}_{F}^{n-1-s}\right]^{A} \in A_{c}^{*}(\mathrm{pt}), a_{l m} \in A_{c}^{*}(\mathrm{pt})$ (Lemma 5.4) and $\sigma_{i}\left(L_{j}\right)=c_{i}^{A}\left(E^{\prime} / E\right)=c_{i}^{A}(-E)$.

Lemma 9.4. For every $s \geq 0, p_{A}\left(\xi^{s}\right) \cdot A_{c}^{*}(X) \subset A_{c}^{*}(X)$.

Proof. Let $f: Y \rightarrow X$ be a projective morphism in $\operatorname{Sm}(F), E^{\prime}=f^{*}(E)$. Consider the Cartesian transverse square

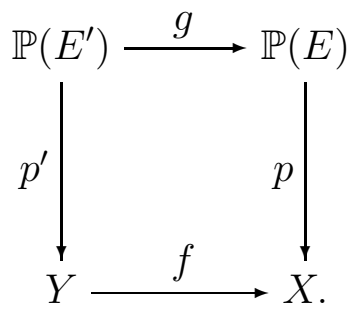

We have

$p_{A}\left(\xi^{s}\right) \cdot f_{A}\left(1_{Y}\right)=p_{A}\left(\xi^{s} \cdot p^{A} f_{A}\left(1_{Y}\right)\right)=p_{A}\left(\xi^{s} \cdot g_{A} p^{\prime A}\left(1_{Y}\right)\right)=p_{A}\left(\xi^{s} \cdot g_{A}(1)\right) \in A_{c}^{*}(X)$ since by Proposition $9.2, \xi^{s} \cdot g_{A}(1) \in A_{c}^{*}(\mathbb{P}(E))$.

Theorem 9.5. For every vector bundle $E$ over $X$ and every characteristic class $c, c^{A}(E) \cdot A_{c}^{*}(X) \subset A_{c}^{*}(X)$.

Proof. Since $c(E)=c^{\prime}(-E)$ for some characteristic class $c^{\prime}$, it is sufficient to prove that $c^{A}(-E) \cdot A_{c}^{*}(X) \subset A_{c}^{*}(X)$ for every $c$. We may assume that

$$
c=c_{\alpha_{1}} c_{\alpha_{2}} \ldots c_{\alpha_{k}}
$$

for some partition $\alpha=\left(\alpha_{1}, \ldots, \alpha_{k}\right)$. Let $a \in A_{c}^{*}(X)$. By Lemma 9.3,

$$
p_{A}\left(\xi^{r-1-\alpha_{1}}\right) \cdot \ldots \cdot p_{A}\left(\xi^{r-1-\alpha_{k}}\right) a=c^{A}(-E) a+\sum_{j>i} a_{j} d_{j}^{A}(E) a \in A^{*}(X),
$$

for some $a_{j} \in A_{c}^{*}(\mathrm{pt})$ and characteristic classes $d_{j}$ of degree bigger than the degree of $c$. By the reverse induction on the degree of $c$, we have $d_{j}^{A}(E) a \in$ $A_{c}^{*}(X)$. By Lemma 9.4, the left hand side of (11) also belongs to $A_{c}^{*}(X)$. Hence, $c^{A}(-E) a \in A_{c}^{*}(X)$. 
Corollary 9.6. For every smooth $X$ and every vector bundle $E$ over $X$, the classes $c_{\alpha}^{A}(E)$ belong to $A_{c}^{*}(X)$ for every partition $\alpha$. Moreover, $c_{\alpha}^{A}(E)=0$ if $|\alpha|>\operatorname{dim}(X)$. In particular, if $X$ is projective, the fundamental polynomial $\mathbf{F}_{X}^{A}$ is of degree at most $\operatorname{dim}(X)$.

Proof. The group $A_{c}^{i}(X)$ is trivial if $i>\operatorname{dim}(X)$.

\section{LANDWEBER-NOVIKOV OPERATIONS}

Let $R$ be a commutative ring. Assume that the group scheme $G=\operatorname{Spec} \mathbb{Z}[\mathbf{b}]$ acts on $\operatorname{Spec} R$. The co-morphism of the action we denote by

$$
\theta_{R}: R \longrightarrow R \otimes \mathbb{Z}[\mathbf{b}]=R[\mathbf{b}] .
$$

For every $r \in R$,

$$
\theta(r)=\sum_{\alpha} s_{\alpha}^{R}(r) \otimes b_{\alpha}
$$

for uniquely determined elements $s_{\alpha}^{R}(r) \in R$. We call the group endomorphisms

$$
s_{\alpha}^{R}: R \longrightarrow R
$$

for all partitions $\alpha$ the Landweber-Novikov operations on $R$.

Now consider the natural action of $G$ on $\operatorname{Spec}(\operatorname{Laz})$ (section 8). The corresponding operations $s_{\alpha}^{\text {Laz }}$ we simply denote by $s_{\alpha}$.

Let $\varepsilon:$ Laz $\rightarrow \mathbb{Z}$ be the restriction of the augmentation map $\mathbb{Z}[\mathbf{b}] \rightarrow \mathbb{Z}$.

Lemma 10.1. The composition

$$
\mathrm{Laz} \stackrel{\theta_{\mathrm{Laz}}}{\longrightarrow} \mathrm{Laz} \otimes \mathbb{Z}[\mathbf{b}] \stackrel{\varepsilon \otimes \mathrm{id}}{\longrightarrow} \mathbb{Z}[\mathbf{b}]
$$

coincides with the embedding Laz $\hookrightarrow \mathbb{Z}[\mathbf{b}]$.

Proof. The homomorphism $\theta_{\text {Laz }}$ corresponds to the group law

$$
\exp (\Phi(\log x, \log y))
$$

on Laz $\otimes \mathbb{Z}[\mathbf{b}]$, where $\Phi$ is the universal group law on Laz. The augmentation of $\Phi$ is the additive group law over $\mathbb{Z}$, whence the result.

Denote by

$$
\mu: \mathbb{Z}[\mathbf{b}] \longrightarrow \mathbb{Z}[\mathbf{b}] \otimes \mathbb{Z}[\mathbf{b}]
$$

the co-multiplication ring homomorphism for the group scheme $G$. Let $A^{*}$ be an oriented ring cohomology theory over $F$. Consider the ring homomorphism of cohomology theories

$$
\widetilde{\mu}=\operatorname{id}_{A} \otimes \mu: \widetilde{A}^{*} \longrightarrow \widetilde{\widetilde{A}}^{*} .
$$

Lemma 10.2. For every $X \in \operatorname{Sm}(F)$ and $a \in K_{0}(X)$,

$$
\widetilde{\mu}\left(\mathbf{P}^{A}(a)\right)=\mathbf{P}^{A}(a) \cdot \mathbf{P}^{\widetilde{A}}(a) .
$$

(In the r.h.s. the first term is a polynomial in the $b_{\alpha}^{\prime}$ and the second - in the $b_{\alpha}^{\prime \prime}$.) 
Proof. The co-multiplication $\mu: \mathbb{Z}[\mathbf{b}] \rightarrow \mathbb{Z}[\mathbf{b}] \otimes \mathbb{Z}[\mathbf{b}]=\mathbb{Z}\left[\mathbf{b}^{\prime}, \mathbf{b}^{\prime \prime}\right]$ satisfies

$$
\sum_{i \geq 0} t^{i+1} \mu\left(b_{i}\right)=\sum_{j \geq 0}\left(\sum_{k \geq 0} t^{k+1} b_{k}^{\prime}\right)^{j+1} b_{j}^{\prime \prime} .
$$

By the splitting principle and multiplicativity property (2), we may assume that $a=[L]$, where $L$ is a line bundle. Hence (with $\xi=c_{1}(L)$ ),

$$
\begin{aligned}
\widetilde{\mu}\left(\mathbf{P}^{A}(L)\right) & =\sum_{i \geq 0} \xi^{i} \mu\left(b_{i}\right) \\
& =\sum_{j \geq 0}\left(\sum_{k \geq 0} \xi^{k} b_{k}^{\prime}\right)^{j+1} \xi^{j} b_{j}^{\prime \prime} \\
& =\sum_{j \geq 0} \mathbf{P}^{A}(L)^{j+1} \xi^{j} b_{j}^{\prime \prime} \\
& =\mathbf{P}^{A}(L) \cdot \sum_{j \geq 0} c_{1}^{\widetilde{A}}(L)^{j} b_{j}^{\prime \prime} \\
& =\mathbf{P}^{A}(L) \cdot \mathbf{P}^{\widetilde{A}}(L) .
\end{aligned}
$$

Corollary 10.3. For every projective variety $X \in \operatorname{Sm}(F)$,

$$
\mu\left(\mathbf{F}_{X}^{A}\right)=\mathbf{F}_{X}^{\widetilde{A}}
$$

Proof. We apply Lemma 10.2 for $a=\left[-T_{X}\right]$ :

$$
\mu\left(\mathbf{F}_{X}^{A}\right)=\mu\left(p_{A} \mathbf{P}_{X}^{A}\right)=p_{A} \widetilde{\mu}\left(\mathbf{P}_{X}^{A}\right)=p_{A}\left(\mathbf{P}_{X}^{A} \cdot \mathbf{P}_{X}^{\widetilde{A}}\right)=p_{\widetilde{A}}\left(\mathbf{P}_{X}^{\widetilde{A}}\right)=\mathbf{F}_{X}^{\widetilde{A}} .
$$

We can express the Landweber-Novikov operations in terms of characteristic numbers in $\widetilde{H}$. This is an analog of Novikov's formula [1, Th. I.8.3] with the cobordism theory replaced by its approximation $\widetilde{H}$.

Proposition 10.4. For every projective variety $X \in \operatorname{Sm}(F)$,

$$
s_{\alpha}\left(\mathbf{F}_{X}^{H}\right)=p_{\widetilde{H}} c_{\alpha}^{\widetilde{H}}\left(-T_{X}\right) \in \mathbb{Z}[\mathbf{b}],
$$

where $p: X \rightarrow \mathrm{pt}$ is the structure morphism.

Proof. Consider the following commutative diagram

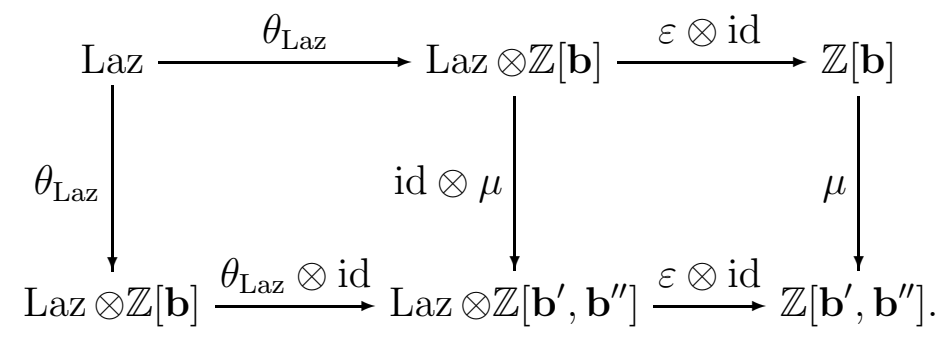


By Lemma 10.1 and Corollary 10.3, the composition $\mu \circ(\varepsilon \otimes \mathrm{id}) \circ \theta_{\text {Laz }}$ takes the class $\mathbf{F}_{X}^{H}$ to

$$
\mathbf{F}_{X}^{\widetilde{H}}=\sum_{\alpha} p_{\widetilde{H}} c_{\alpha}^{\widetilde{H}}\left(-T_{X}\right) b_{\alpha}^{\prime \prime}
$$

By Lemma 10.1, another composition $(\varepsilon \otimes \mathrm{id}) \circ\left(\theta_{\mathrm{Laz}} \otimes \mathrm{id}\right) \circ \theta_{\mathrm{Laz}}$ takes $\mathbf{F}_{X}^{H}$ to

$$
\sum_{\alpha} s_{\alpha}\left(\mathbf{F}_{X}^{H}\right) b_{\alpha}^{\prime \prime}
$$

\section{INVARIANT IDEALS}

Let $R$ be a commutative ring. Assume that the group scheme $G=\operatorname{Spec} \mathbb{Z}[\mathbf{b}]$ acts on Spec $R$. An ideal $I \subset R$ is called invariant if $s_{\alpha}^{R}(I) \subset I$ for every $\alpha$.

Let $p$ be a prime integer. The ideal $p \mathbb{Z}[\mathbf{b}]$ in $\mathbb{Z}[\mathbf{b}]$ is obviously prime and invariant with respect to the action of $G$ on itself by left translations. Therefore, the intersection $I(p)=\operatorname{Laz} \cap p \mathbb{Z}[\mathbf{b}] \subset$ Laz is a prime invariant ideal in Laz.

Let $n=0,1,2, \ldots, \infty$. Denote by $I(p, n)$ the ideal in $I(p)$ generated by all $a \in I(p)$ of degree $\leq p^{n}-1$. For example, $I(p, 0)=p$ Laz and $I(p, \infty)=I(p)$.

Thus, for every prime $p$ we have a chain of prime invariant ideals in Laz:

$$
p \operatorname{Laz}=I(p, 0) \subset I(p, 1) \subset \cdots \subset I(p, n) \subset \cdots \subset I(p, \infty)=I(p) .
$$

It is known (see [9, Prop. VII.4.21] and [5, Th. 2.7]) that every ideal $I(p, n)$ is prime and invariant and the only nonzero prime invariant ideals in Laz are $I(p, n)$ for all prime $p$ and $n \geq 0$.

Let $X$ be a projective smooth variety over a field $F$. The set

$$
I(X)=\left\{\mathbf{F}_{Y}^{H} \in \operatorname{Laz} \text { for all } Y \text { such that } \operatorname{Mor}_{F}(Y, X) \neq \emptyset\right\}
$$

is a graded ideal in Laz. Let $q: X \rightarrow$ pt be a structure morphism. For every projective morphism $f: Y \rightarrow X$,

$$
q_{\widetilde{H}} f_{\widetilde{H}}\left(1_{Y}\right)=(q f)_{\widetilde{H}}\left(1_{Y}\right)=\mathbf{F}_{Y}^{H}
$$

Hence

$$
I(X)=q_{\widetilde{H}} \widetilde{H}_{c}(X)
$$

Recall that $n_{X}$ is the gcd of $\operatorname{deg}(x)$ over all closed points $x$ of a variety $X$.

Example 11.1. $I(X)_{0}=n_{X} \mathbb{Z}$. If $X(F) \neq \emptyset, I(X)=$ Laz.

Theorem 11.2. For a projective variety $X \in \operatorname{Sm}(F)$ over an infinite field $F$, the ideal $I(X) \subset$ Laz is invariant.

Proof. Let $f: Y \rightarrow X$ be a morphism, $q: X \rightarrow$ pt the structure morphism. By Proposition 10.4 and Corollary 9.6,

$$
s_{\alpha}\left(\mathbf{F}_{Y}^{H}\right)=q_{\widetilde{H}} f_{\widetilde{H}}\left(c^{\widetilde{H}}\left(-T_{Y}\right)\right) \in q_{\widetilde{H}}\left(\widetilde{H}_{c}(X)\right)=I(X) .
$$


Let $P$ be a minimal prime ideal in Laz containing $I(X)$. By [6, Th. 3.1], $P$ is invariant and hence $P=I(p, n)$ for some prime integer $p$ and $n=0,1, \ldots, \infty$. Clearly, $P$ is the only minimal prime ideal containing $I(X)$ and $p$. We set $n_{p}(X)=n$. If for a prime integer $p$ there is no invariant prime ideal containing $I(X)$ and $p$, we set $n_{p}(X)=\infty$. Thus, for every projective variety $X$ we have the numbers $n_{p}(X)$ assigned for each prime integer $p$.

Proposition 11.3. Let $X \in \operatorname{Sm}(F)$ be a projective variety, $p$ a prime integer. Then the following conditions are equivalent:

(1) $p \mid n_{X}$;

(2) There exists an invariant prime ideal of Laz containing $I(X)$ and $p$.

Proof. If $I\left(p, n_{p}(X)\right)$ is the minimal prime ideal, then $I(X)_{0} \subset p \mathbb{Z}$, i.e. $p \mid n_{X}$. Conversely, let $p \mid n_{X}$. Since the radical of $I(X)$ is the intersection of all minimal ideals, one of the minimal ideals contains $p$.

Proposition 11.4. Let $X$ and $Y$ be projective smooth varieties such that $\operatorname{Mor}(Y, X) \neq \emptyset$. Then $n_{p}(Y) \leq n_{p}(X)$ for every prime $p$.

Proof. We have $I(Y) \subset I(X) \subset I\left(p, n_{p}(X)\right)$. The minimal prime ideal between $I(Y)$ and $I\left(p, n_{p}(X)\right)$ is equal $I\left(p, n_{p}(Y)\right)$, hence $n_{p}(Y) \leq n_{p}(X)$.

\section{REFERENCES}

[1] J. F. Adams, Stable homotopy and generalised homology, University of Chicago Press, Chicago, IL, 1995, Reprint of the 1974 original. MR 96a:55002

[2] W. Fulton, Intersection theory, Springer-Verlag, Berlin, 1984.

[3] R. Hartshorne, Algebraic geometry, Springer-Verlag, New York, 1977, Graduate Texts in Mathematics, No. 52. MR 57 \#3116

[4] M.-A. Knus, A. Merkurjev, M. Rost, and J.-P. Tignol, The book of involutions, American Mathematical Society, Providence, RI, 1998, With a preface in French by J. Tits.

[5] P. S. Landweber, Annihilator ideals and primitive elements in complex bordism, Illinois J. Math. 17 (1973), 273-284. MR 48 \#1235

[6] _ Associated prime ideals and Hopf algebras, J. Pure Appl. Algebra 3 (1973), 43-58. MR 49 \#10678

[7] M. Levine and F. Morel, Cobordisme algébrique I, C. R. Acad. Sci. Paris Sér. I Math. 332 (2001).

[8] I. Panin and A. Smirnov, Push-forwards in oriented cohomology theories of algebraic varieties, http://www.math.uiuc.edu/K-theory/0459/ (2000).

[9] Y. B. Rudyak, On Thom spectra, orientability, and cobordism, Springer-Verlag, Berlin, 1998, With a foreword by Haynes Miller. MR 99f:55001

[10] R. E. Stong, Notes on cobordism theory, Princeton University Press, Princeton, N.J., 1968, Mathematical notes.

Alexander Merkurjev, Department of Mathematics, University of CaliforNiA, Los Angeles, CA 90095-1555, USA

E-mail address: merkurev@math.ucla.edu 